\title{
Erratum to: Is pre-operative haemoglobin A1c level a successful predictor of adverse outcome after cardiac surgery?
}

\author{
Ai Hooi Tee ${ }^{1}$, R. Hasan², K. E. Mclaughlin², D. J. M. Keenan² and S. Datta ${ }^{2 *}$
}

\section{Erratum}

After the publication of this Meeting Abstract [1] it was noticed that the author name "Ai Hooi Tee" was misspelled as "Ai Hooe Tee".

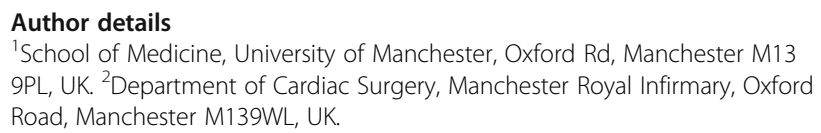

\section{Reference}

1. Tee AH, Hasan R, Mclaughlin KE, Keenan DJM, Datta S. Is pre-

operative haemoglobin A1c level a successful predictor of adverse outcome after cardiac surgery? J Cardiothorac Surg. 2015;10 Suppl 1: A328. doi:10.1186/1749-8090-10-S1-A328.

\footnotetext{
* Correspondence: ivytee25@gmail.com

${ }^{2}$ Department of Cardiac Surgery, Manchester Royal Infirmary, Oxford Road, Manchester M139WL, UK
} 